\title{
Analyses de diagnostic rapide en immunologie
}

\author{
Caroline Polia,b, Céline Beauvillain ${ }^{\mathrm{a}, \mathrm{b}}$, Pascale Jeannin ${ }^{\mathrm{a}, \mathrm{b}}$, Gilles Renier ${ }^{\mathrm{a}, \mathrm{c}}$, Alain Chevaillera,a,
}

\section{Résumé}

Certaines pathologies, relevant d'un dysfonctionnement de la réponse immunitaire, peuvent rapidement compromettre le pronostic fonctionnel de certains organes, voire le pronostic vital, car le plus souvent ce sont des maladies systémiques, de diagnostic difficile au début quand elles sont paucisymptomatiques alors qu'elles peuvent flamber subitement. C'est pourquoi le laboratoire d'immunologie, aux techniques encore non automatisées pour certaines, et qui travaille majoritairement en série, se doit de bousculer son organisation pour répondre à la demande argumentée d'un clinicien confronté à une urgence diagnostique. II s'agit de la recherche d'auto-anticorps dans le cadre des glomérulonéphrites rapidement progressives et des syndromes pneumo-rénaux, du dosage des médiateurs de l'allergie dans le contexte des chocs anaphylactiques, du diagnostic de l'infection tuberculeuse latente en pédiatrie, de la recherche des lymphocytes T activés en cas de syndrome d'activation macrophagique et enfin de l'analyse qualitative et quantitative des immunoglobulines, notamment devant une suspicion de syndrome d'hyperviscosité ou d'insuffisance rénale aiguë d'origine myélomateuse.

ANCA - anticorps anti-MBG - glomérulonéphrite rapidement progressive - syndrome pneumorénal - choc anaphylactique - histamine - tryptase infection tuberculeuse latente - test IGRA - lymphocytes T activé - syndrome d'activation macrophagique - syndrome d'hyperviscosité.

\section{Introduction}

Cet article se veut le fruit de l'expérience d'un laboratoire d'immunologie et d'allergologie de CHU. II présentera les indications de demandes de diagnostic immunologique rapide, à l'exclusion du typage HLA dans un contexte de transplantation, qui a lui seul justifie un chapitre de livre [1]. Pourquoi «diagnostic rapide» et non pas «d'urgence» ? Pour deux raisons: i) la première sémantique et réglementaire: dans très peu d'établissements existent une garde ou une astreinte biologique permettant la réalisation 24/24, 7 jours sur 7 , de ces analyses. Afficher de tels examens «d'urgence»

a Laboratoire d'immunologie et d'allergologie

Plateau de biologie hospitalière (PBH)

Institut de biologie en santé (IBS)

Centre hospitalier universitaire d'Angers

4, rue Larrey

49933 Angers cedex 9

b Inserm U892/CNRS 6299 - CRCNA - Université d'Angers

49933 Angers cedex 9

c Université d'Angers - UPRES EA 3142

49933 Angers cedex 9

${ }^{*}$ Correspondance

AIChevailler@chu-angers.fr

article reçu le 22 mai, accepté le 11 juin 2015

(C) 2015 - Elsevier Masson SAS - Tous droits réservés.

\section{SUMmarY}

\section{Immunological emergency diagnosis assays}

Some diseases with immune response dysfunction could rapidly lead to some organs failure, even could compromise the vital prognosis. Because they are pauci-symptomatic at the beginning, the diagnosis of those systemic diseases is often very difficult, whereas they could rapidly burst out. So, immunology laboratory with its even non automatized techniques and usually programmed serial immunoassays, must revised its works schedule to realize emergency test to respond to the physician's arguments in front of a clinical emergency. The diseases in question are rapidly progressive glomerulonephritis and pneumorenal syndrome, acute anaphylaxy reaction, latent tuberculosis, hemophagocytic syndrome and hyperviscosity syndrome and myelomatous acute renal failure.

ANCA - GBM antibody - rapidly progressive glomerulonephritis - pneumorenal syndrome - histamine - tryptase - acute anaphylaxy reaction - activated T lymphocytes - hemophagocytic syndrome - hyperviscosity syndrome - myelomatous acute renal failure.

serait tendre des verges au Cofrac pour se faire battre; ii) et, pour être provocateur, disons qu'il n'y a pas d'examens immunologiques urgents, il n'y a que des cliniciens pressés, qui le plus souvent ignorent les principes des méthodes diagnostiques utilisées et le mode organisationnel du laboratoire (dosages en flux continu ou en série notamment). Bien entendu un tel aphorisme est outrancier, mais veut pointer la nécessité du dialogue clinico-biologique, seul à même d'apporter une plus-value au seul résultat brut. Pour exemple, l'appel récent d'un jeune interne, un après-midi de semaine à 17 heures alors que les techniciens étaient sur le départ, pour une demande d'ANCA (antineutrophil cytoplasmic antibodies) en «urgence » et qui s'est étonné que vu l'heure tardive la procédure (décrite ci-dessous) ne soit pas réalisable, s'imaginant que «comme tout le reste, ce devait être de la biologie presse-bouton " (sic) (seul le multiplexage a été réalisé).

Seront donc abordées successivement les situations où le diagnostic, aux heures ouvrables du laboratoire en semaine, relève d'une urgence vraie ou organisationnelle (veille de grand week-end par exemple). II s'agit de la recherche d'auto-anticorps dans le cadre des glomérulonéphrites rapidement progressives et des syndromes pneumo-rénaux, le dosage des médiateurs de l'allergie dans le contexte des chocs anaphylactiques, le diagnostic de l'infection tuberculeuse latente (ITL) en pédiatrie, la recherche des lymphocytes T activés en cas de syndrome d'activation macrophagique (SAM) et enfin l'analyse qualitative 
et quantitative des immunoglobulines, notamment devant une suspicion de syndrome d'hyperviscosité ou d'insuffisance rénale aiguë (IRA) d'origine myélomateuse.

\section{Anticorps anti-cytoplasme des polynucléaires neutrophiles et anticorps anti-membrane basale glomérulaire}

\subsection{Contexte de demande de diagnostic rapide}

Les ANCA (antineutrophil cytoplasmic antibodies) et les anticorps anti-membrane basale glomérulaire (MBG) sont demandés en urgence par le clinicien en cas de glomérulonéphrite rapidement progressive mettant en jeu le pronostic fonctionnel voire vital du patient, ou encore en cas d'hémorragie intra-alvéolaire massive.

La rapidité d'initiation du traitement est primordiale afin de limiter les dommages d'organe, voire préserver le pronostic vital. Les anticorps anti-cytoplasme des polynucléaires neutrophiles (ANCA) sont dirigés contre les protéines des granules des neutrophiles et des lysosomes des monocytes, et leur présence est associée à la polyangéite avec granulomatose, la polyangéite microscopique, et la granulomatose éosinophilique avec polyangéite [2]. Les deux antigènes cibles dont les auto-anticorps sont associés à une forte valeur prédictive positive (VPP) et présentant donc un intérêt diagnostique en urgence dans la vascularite à ANCA sont la protéinase 3 (PR3) et la myéloperoxydase (MPO). Les anticorps anti-PR3 et anti-MPO sont classiquement associés à une fluorescence de type cytoplasmique (c-ANCA) et périnucléaire ( $p-A N C A)$, respectivement, sur lame de polynucléaires neutrophiles humains fixés à l'éthanol.

La vascularite avec anticorps anti-MBG (anciennement syndrome de Goodpasture) est associée à la présence d'autoanticorps dirigés contre la chaine alpha 3 du collagène de type IV.

\subsection{Tests commercialisés, interprétation biologique}

Dans un tel contexte, la décision de mettre en route un traitement immunosuppresseur lourd et non dénué d'effets secondaires, mais seul à même de préserver le pronostic, ne peut attendre la confirmation diagnostique apportée par l'examen anatomopathologique de l'organe biopsié, le rein le plus souvent [3]. La recherche des ANCA, habituellement exécutée par séries programmées, doit alors se faire dans la journée, en associant immunofluorescence indirecte (IFI) et test en phase solide, immunodot, fluorométrie ou multiplexage (ou ALBIA pour adressable laser bead immunoassays), mieux adaptés à une demande ponctuelle que l'ELISA en microplaque [4].

Un prélèvement sur tube sec sera acheminé à température ambiante, puis centrifugé pour dépister les auto-anticorps sur sérum. Le résultat biologique est rendu dans un délai maximum de 24 heures [5].

Concernant les ANCA, la démarche de dépistage est classiquement en 2 étapes: un test d'IFI sur lames de polynucléaires neutrophiles avec au moins deux types de fixateurs (éthanol et formol), complété en cas de positivité (c-ANCA ou p-ANCA) par un test en phase solide recherchant les spécificités d'autoanticorps anti-MPO, anti-PR3.

Les différentes techniques de tests en phase solide sont I'ELISA, ALBIA, dots, FEIA (fluorescent-enzyme-immunoassay ou fluorométrie) et CLIA (chimioluminescence).

Certains fournisseurs proposent une solution spécifiquement adaptée au contexte de diagnostic rapide proposant un seul test combinant le dépistage par immunofluorescence des p-ANCA et c-ANCA, ainsi que la spécificité anti-MPO ou anti-PR3, soit par IFI couplée à des dots (Euroimmun) [6], soit par IFI couplée à des microbilles (Generic assay) [7]. La sensibilité et spécificité de l'IFI combinée aux tests en phase solide est excellente dans le cadre d'un diagnostic initial des vascularites à ANCA dans un contexte d'urgence: 83,3 et $100 \%$, respectivement [3].

Cependant, la présence d'ANCA peut également être associée à d'autres situations pathologiques telles que la polyarthrite rhumatoïde, ou les endocardites infectieuses $[8,9]$.

De plus la granulomatose éosinophilique avec polyangéite est dans environ un cas sur deux associée à une séronégativité [10].

II est parfois difficile de poser le diagnostic de vascularite associée aux ANCA (AAV), témoin ce cas clinique que nous avons rapporté [11]. II s'agissait d'une patiente, recevant une immunosuppression pour sa polyarthrite rhumatoïde, ayant développé une AAV dont la réponse autoanticorps était restreinte par les effets des corticoïdes et du méthotrexate [10], n'étant objectivée en IFI que sur les polynucléaires fixés par le formol et que dans un seul des 4 tests en phase solide. Les messages à retenir sont les suivants: i) pour l'IFI, il faut impérativement associer des frottis de PNN fixés par l'éthanol et le formol; dans le cas présent, la seule recherche avec des frottis fixés par l'éthanol aurait conclu à la négativité; ii) pour les tests en phase solide, au moindre doute, qui ne peut provenir que de la clinique, d'où l'importance du dialogue clinico-biologique, il ne faut pas hésiter à les multiplier: dans le cas présent, c'est bien parce que, en association avec un résultat suspect d'IFI, le résultat du premier test réalisé (ALBIA) n'était pas totalement négatif que l'enquête a été poursuivie. Concernant les anticorps anti-MBG, la méthode de référence est l'IFI sur coupe de rein de primate. Les tests en phase solide dépistant les spécificités MPO et PR3 des ANCA intègrent généralement également la spécificité MBG. Parmi les principaux écueils, I'IFI peut manquer de sensibilité à des titres faibles d'anticorps et nécessite une expertise de lecture. De plus, en début de maladie, une phase dite séronégative a été décrite, attribuée à la fixation des anticorps dans le rein. Ces tests sont prescrits dans le cadre d'un dialogue clinicobiologique permettant d'éviter les différentes sources d'erreur décrites ci-dessus et d'assurer une réponse rapide, assurant une prise en charge thérapeutique dans les plus brefs délais.

\section{Histamine et tryptase}

\subsection{Contexte de demande de diagnostic rapide}

L'histamine, ou 2- (4-imidazolyl) éthylamine et la tryptase sont des médiateurs préformés impliqués dans l'anaphylaxie, définie comme une réaction d'hypersensibilité immédiate grave potentiellement fatale $[12,13]$. 
L'anaphylaxie est stratifiée en 4 stades de gravité. II s'agit d'une urgence vitale en raison de la rapidité d'installation et d'aggravation des symptômes, aboutissant en l'absence d'intervention médicale appropriée à un choc hypovolémique voire un arrêt cardio-respiratoire et au décès du patient; ce qui explique que l'urgence ne porte que sur la phase préanalytique [14], le praticien n'attendant pas le résultat du laboratoire pour la mise en route du traitement salvateur (adrénaline).

Sur le plan biologique, le taux plasmatique de l'histamine est corrélé à la sévérité des manifestations cardio-pulmonaires de l'anaphylaxie [15]. À noter la bonne valeur prédictive positive de la tryptase pour l'anaphylaxie. De plus, il a été montré que plus la réaction anaphylactique est sévère, plus le taux de tryptase est élevé, l'augmentation du taux de tryptase étant inversement corrélée à la chute de pression artérielle [16].

Parmi les cellules capables de synthétiser de l'histamine, les mastocytes et les basophiles se distinguent par leur capacité à stocker l'histamine dans leurs granules, permettant un relargage massif et immédiat lors du pontage des lgE spécifiques présentes à la surface des basophiles et mastocytes des sujets sensibilisés. La tryptase est actuellement considérée comme le meilleur marqueur de la dégranulation mastocytaire. II n'existe pas de tryptase dans les basophiles. La tryptase est une enzyme tétramérique contenue dans les granules des mastocytes avec l'histamine qui est libérée avec elle lors de la dégranulation. La tryptase béta est uniquement présente dans les granules, tandis que l'alpha-protryptase est sécrétée faiblement de manière continue [13].

Les dosages d'histamine et de tryptase permettent de conforter le diagnostic d'anaphylaxie et donc l'origine allergique des manifestations cliniques. II en résulte une contre-indication définitive de la substance responsable afin d'éviter une récidive, ainsi qu'une indication à une désensibilisation lorsque cette dernière est disponible, selon l'allergène concerné (ex: venin d'hyménoptères). Dans cet objectif, des examens immédiats et une consultation spécifique d'allergologie à distance sont à prévoir de façon impérative.

\subsection{Tests commercialisés, interprétation biologique}

Les méthodes de dosages de l'histamine commercialisées actuellement disponibles aux normes IVD (in vitro diagnostic use) sont d'une part la technique par radio-immunoanalyse (RIA) (Beckman Coulter, Immunotech) et d'autre part des techniques immuno-enzymatiques (EIA) (Beckman Coulter, Immunotech et Demeditec). L'histamine est normalement présente en quantité très faible dans le plasma $(<1 \mathrm{nM})$. Le seuil de positivité de ces kits est généralement à 10nM. En dehors d'une hémolyse, l'augmentation plasmatique de l'histamine est attribuée à une réaction anaphylactique. L'histamine est rapidement métabolisée in vivo par l'histamine $\mathrm{N}$-methyltransférase (HNMT) et la diamine oxydase (DAO) d'où une demie vie courte d'environ 1 minute. Le prélèvement plasmatique pour le dosage de l'histamine est ainsi réalisé dans les 30 minutes suivant le début des symptômes. Un autre prélèvement est généralement réalisé à 1 heure du prélèvement, en parallèle du premier prélèvement pour le dosage de la tryptase. Le prélèvement de sang sur EDTA est acheminé dans les plus brefs délais au laboratoire (indication précise des horaires de la réaction indispensable) où il est immédiatement centrifugé avec congélation immédiate du plasma. Il faut savoir interpréter les valeurs mesurées d'histamine en fonction des circonstances cliniques, car il existe des faux négatifs (fin de grossesse, traitement héparinique lors de circulation extracorporelle).

Le dosage de la tryptase est réalisé par une technique immunofluorométrique de type sandwich commercialisée par le laboratoire Phadia Thermofisher.

Concernant la tryptase, son pic maximal est atteint dans le sang environ $1 \mathrm{~h}$ après le déclenchement du choc. Elle disparaît du sérum en 10 à $12 \mathrm{~h}$. Dans les chocs graves elle peut persister à des concentrations élevées, plusieurs heures à plusieurs jours après la mort, ce qui explique la possibilité de demande de dosages de la tryptase dans le cadre d'une autopsie [17]. Le prélèvement sanguin sera réalisé sur tube sec, EDTA ou héparine. II est conseillé de réaliser au moins deux prélèvements: I'un réalisé 30 minutes à 2 heures après l'événement clinique et un deuxième 24 à $48 \mathrm{~h}$ après. Ce deuxième prélèvement permet d'avoir le niveau basal pour l'individu et représente une précieuse aide à l'interprétation biologique du premier tube. Le prélèvement sera acheminé à température ambiante si transport rapide. Le cas échéant il sera réfrigéré. Le prélèvement peut être conservé au laboratoire pendant 3 jours au réfrigérateur, ou au congélateur pour une durée prolongée.

\section{Interferon gamma release assay (IGRA)}

\subsection{Contexte de demande de diagnostic rapide}

Les tests de relargage de l'IFN- $\gamma$ permettent de mettre en évidence la présence de lymphocytes $T$ mémoires spécifiques de certains antigènes de Mycobacterium tuberculosis (ESAT-6, CFP-10 et plus ou moins TB7.7). II existe de rares positifs en réponse à d'autres mycobactéries exprimant également ces antigènes: Mycobacterium kansasii, Mycobacterium marinum et Mycobacterium szulga [18].

L'intérêt principal de ces tests est de dépister les sujets ayant une infection tuberculeuse latente (ITL). Cependant la demande de test IGRA en urgence concerne le plus souvent l'aide diagnostique de tuberculose latente ou active chez les enfants.

Les indications des tests IGRA ont été définies par le Haut Conseil de Santé publique (HCSP) en 2011 comme étant [19]: - avant la mise en route d'un traitement par anti-TNF-alpha, - au cours d'une enquête autour d'un cas index,

- à l'embauche pour le personnel professionnellement exposé,

- au cours du bilan initial des patients infectés par le VIH.

Si l'on s'intéresse plus précisément au cadre pédiatrique, il est stipulé que les indications de prescription d'un test IGRA sont:

- enquête autour d'un cas: «chez l'enfant à partir de l'âge de 5 ans: I'un ou l'autre des tests IDR ou IGRA peut être utilisé»;

"Les tests IGRA peuvent être utilisés comme aide au diagnostic des tuberculoses maladies du jeune enfant en complément des autres investigations "; 
- pour les enfants migrants âgés de 5 à 15 ans, le dépistage d'ITL « pourrait être pratiqué avec un test IGRA ».

Le diagnostic de la tuberculose chez l'enfant est un problème majeur. Ces derniers sont beaucoup plus à risque d'évolution de tuberculose latente à active (40\% dans l'année qui suit le contage pour les nourrissons versus $10 \%$ dans les 2 ans pour les adultes). Ils présentent plus fréquemment des formes disséminées (méningite tuberculeuse), avec des sites cliniques peu spécifiques et des formes pulmonaires peu bacillifères rendant les examens bactériologiques moins performants (microbiologie mise en défaut dans la moitié des cas), en plus de la difficulté de réalisation de ces tests chez le jeune enfant. Le diagnostic de tuberculose maladie chez l'enfant ne repose donc pas uniquement sur la microbiologie mais résulte d'un faisceau d'argument [20]. L'IDR est le seul test recommandé chez les enfants de moins de 5 ans. II n'existe pas actuellement de recommandation pour l'IGRA chez l'enfant de moins de 5 ans.

Les réserves s'expliquent par une sensibilité moindre de tous les tests immunologiques avant 5 ans [21].

À l'heure actuelle les publications ont montré des résultats disparates avec des cohortes très hétérogènes, ne permettant pas de conclure pour le moment. À noter que la prescription conjointe de la CRP peut être intéressante car elle permet en cas de résultat indéterminé de plutôt s'orienter vers une autre pneumopathie. De plus un nouveau prélèvement après antibiothérapie peut être envisagé pour écarter une tuberculose maladie sous-jacente.

Selon les données de l'OMS 2014, les IGRA ont une valeur pronostique et une VPP équivalente à l'IDR, si l'on considère l'apparition d'une tuberculose active chez des sujets non traités.

De plus, une méta-analyse a mis en évidence une meilleure spécificité de l'IGRA par rapport à l'IDR, avec une sensibilité à peu près similaire [22].

\subsection{Tests commercialisés, interprétation biologique}

Les tests IGRA actuellement disponibles sont le Quantiféron $^{\circledR}$ (Qiagen) et le T-SPOT TB ${ }^{\circledR}$ (Oxford Immunotec) [23].

Concernant le Quantiféron ${ }^{\circledR}$, le sang est prélevé directement sur 3 tubes test: un tube «nul» permettant d'évaluer la sécrétion basale d'IFN- $\gamma$, un tube "antigène » évaluant la réponse mémoire spécifique et un tube « mitogène » avec la phytohémagglutinine $\mathrm{A}(\mathrm{PHA})$. (Le sang peut également être prélevé dans un seul tube contenant de l'héparine de lithium comme anticoagulant avant d'être transféré vers les tubes Quantiféron ${ }^{\circledR}$.) Le prélèvement doit être immédiatement agité 10 fois pour décrocher le mitogène ou les antigènes. L'absence d'agitation peut entraîner un résultat ininterprétable par absence de réponse du mitogène. Les tubes doivent être acheminés au laboratoire rapidement (dans un délai maximal de 12 heures) puis les tubes sont incubés 16 à $24 \mathrm{~h}$ à $37^{\circ} \mathrm{C}$. Les tubes sont ensuite centrifugés, le plasma est décanté et la quantité d'IFN- $\gamma(\mathrm{Ul} / \mathrm{mL})$ est mesurée par ELISA. Ce dernier peut être réalisé par technique manuelle ou automatisée. Le résultat du tube nul est soustrait à celui du tube antigène pour ne garder que la sécrétion d'IFN- $\gamma$ spécifiques des antigènes ESAT-6, CFP10. Le tube contrôle positif permet de vérifier la capacité des lymphocytes à sécréter une quantité détectable d'IFN- $\gamma$. Un tube positif inférieur à $0,5 \mathrm{Ul} / \mathrm{mL}$ rend le test ininterprétable. En dehors d'un défaut d'agitation des tubes post-prélèvement la cause la plus fréquente en est l'existence d'une lymphopénie. L'interprétation du test est qualitative à partir de données chiffrées.

Une zone de difficulté d'interprétation du test autour du seuil est définie dans la littérature de 0,3 à $0,7 \mathrm{UI} / \mathrm{mL}$ d'IFN- $\gamma$ [24]. Comme pour tout ELISA, une hémolyse ou lipémie importante peuvent constituer une interférence.

Une nouvelle version du test, le Quantiféron Plus ${ }^{\circledR}$ comporte un tube antigène supplémentaire intégrant des antigènes peptidiques courts spécifiques de LT CD8+ spécifiques de Mycobacterium tuberculosis, en plus des antigènes peptidiques longs ciblant les LT CD4+ mémoires spécifiques. Ce nouveau test pourrait améliorer la valeur prédictive du test, en particulier dans le cadre de la tuberculose maladie. En particulier, les lymphocytes T CD8+ spécifiques de Mycobacterium tuberculosis ont été détectés chez les sujets atteints de la tuberculose active présentant une co-infection au VIH, ainsi que chez les enfants atteints de la tuberculose maladie [25-27]. De plus il pourrait permettre de diminuer le nombre de résultats ininterprétables chez les patients immunodéprimés. Concernant le T-SPOT. TB ${ }^{\circledR}$, le sang est prélevé sur tube hépariné. L'acheminement du prélèvement est à température ambiante. L'échantillon sera traité dans les 8 heures. Pour les délais plus importants il existe un stabilisant permettant de porter le délai de conservation à 32 heures. Après isolement des cellules mononucléées par gradient de densité, les cellules sont déposées dans des puits recouverts d'anticorps anti-IFN $\gamma$. II y a un puits contrôle négatif (milieu seul), un puits contrôle positif avec la PHA et deux puits antigène contenant des peptides de $M$. tuberculosis. Les puits sont incubés 16 à 20 h à $37^{\circ} \mathrm{C}$ puis lavés et incubés avec l'anticorps secondaire anti-IFN $\gamma$ conjugué à la phosphatase alcaline. Chaque spot correspond à un clone lymphocytaire T mémoire spécifique des peptides de $M$. tuberculosis.

L'interprétation du test est qualitative à partir de données chiffrées.

Ce test nécessite une expérience technique de manipulation et de culture cellulaire. De façon similaire au Quantiféron ${ }^{\circledR}$, l'interprétation du T-SPOT.TB ${ }^{\circledR}$ comporte une zone de difficulté définie dans la littérature entre 6 et 10 spots.

\section{5. Évaluation de l'expression des molécules HLA-DR sur les lymphocytes T}

\subsection{Contexte de demande de diagnostic rapide}

Dans le domaine de l'immunologie cellulaire, les cliniciens, et notamment les pédiatres formés à l'école du Centre de reférence des déficits immunitaires héréditaires (CEREDIH) peuvent faire appel au laboratoire pour obtenir dans un délai limité une évaluation de l'activation des lymphocytes $\mathrm{T}$ dans le cadre des syndromes dits d'activation lymphohistiocytaire, ou hémophagocytaires ou d'activation macrophagique [28].

Ces syndromes qui engagent le pronostic vital en provoquant une défaillance multiviscérale, correspondent 
en effet à un état d'activation incontrôlée du système immunitaire et en particulier des cellules T et des macrophages. Ils peuvent survenir dans des contextes étiologiques variés qu'il est pour la plupart possible de rassembler schématiquement dans quelques cadres. Les formes acquises sont les plus fréquentes et surviennent chez l'adulte ou l'adolescent. dans des contextes d'infection, de néoplasie, notamment des lymphomes, de maladie systémique ou d'immunodépression. Les formes héréditaires peuvent être de révélation précoce chez l'enfant ou plus tardive. Elles sont dues à une anomalie d'un gène, tel que celui de la perforine, qui rend inefficace la cytotoxicité des cellules T CD8+ et NK; le système s'engage alors dans un cercle vicieux d'amplification d'une réponse inopérante [28]. Le diagnostic doit être évoqué devant toute cytopénie fébrile et repose sur un faisceau d'arguments cliniques et biologiques; et pour les formes pédiatriques, des critères ont été émis par l'Histiocytes Society et révisés en 2004 (tableau I) [29]. Les choses sont moins bien codifiées pour l'adulte.

D'autres éléments pourraient également être pris en compte comme l'élévation de nombreuses cytokines comme le $\mathrm{TNF}-\alpha$; et, en dépit des dénominations du syndrome, l'hémophagocytose n'est ni pathognomonique ni constante.

\subsection{Tests commercialisés, interprétation biologique}

Bien qu'elle ne figure pas dans les critères énoncés en 2004, l'évaluation de l'activation des lymphocytes T par mesure de l'expression des molécules HLA-DR sur leur membrane, est une analyse simple à mettre en œuvre pour n'importe quel laboratoire disposant d'un cytomètre en flux; et son résultat peut être obtenu rapidement, pour peu que le laboratoire soit prévenu et que l'échantillon lui parvienne sans retard. II suffit en outre de compléter le très basique marquage CD3/CD4/CD8 avec un antisérum anti-HLA-DR conjugué à un quatrième fluorochrome, pour pouvoir préciser le degré d'activation de chacune des sous-populations $\mathrm{T}$, principalement CD3+CD4+ CD8- et CD3+CD8+ CD4-. On peut aussi garder en mémoire que dans ce test les lymphocytes non T exprimant HLA-DR sont un reflet de la population des cellules $B$.

Tableau I - Critères diagnostiques

du syndrome d'activation macrophagique: 5 critères sur les 8 sont requis.

\footnotetext{
1. Fièvre $\geq 38,5^{\circ} \mathrm{C}$

2. Splénomégalie

3. Bi-cytopénie (hémoglobine $<9 \mathrm{~g} / \mathrm{dL}$, plaquettes $<100 \mathrm{G} / \mathrm{L}$, neutrophiles $<1000 / \mathrm{mm}^{3}$ )

4. Taux élevé de triglycérides $(>3,0 \mathrm{mmol} / \mathrm{L})$

ou fibrinogène bas $(<1,5 \mathrm{~g} / \mathrm{dL})$

5. Ferritine élevée (> $500 \mathrm{ng} / \mathrm{mL}$ )

6. Image d'hémophagocytose (moelle, rate, ganglion, foie)

7. Diminution de la cytotoxicité des cellules NK

8. Taux élevé de CD-25 soluble (>2400 UI/mL)
}

D'après [29].
Cette analyse est généralement encore plus simple à réaliser sur du liquide céphalo-rachidien, puisqu'il n'est habituellement pas besoin de se débarrasser des hématies pour «voir» les lymphocytes. La présence de quelques milliers de cellules dans l'échantillon peut suffire pour répondre. Il convient cependant de garder à l'esprit qu'il est alors absolument indispensable d'étudier le sang périphérique en parallèle.

Comme le montrent les figures 1 et 2, ce que l'on recherche est l'observation d'une activation massive des cellules $T$ CD8+ accompagnant une augmentation de leur nombre, responsable d'une inversion du rapport T CD4+ CD8- / T CD4- CD8+. À noter qu'il existe physiologiquement un petit pourcentage, ne dépassant pas $10 \%$ habituellement, de lymphocytes T exprimant HLA-DR à leur membrane. L'exploration apporte un argument supplémentaire, à confronter avec les autres éléments cliniques et biologiques au moment du diagnostic et de la décision thérapeutique. Elle permet également de suivre l'évolution et le retour vers les équilibres physiologiques.

\section{Analyse qualitative et quantitative rapide des immunoglobulines}

\subsection{Contexte de demande de diagnostic rapide}

Une immunoglobuline monoclonale se caractérise par l'augmentation sélective d'une seule espèce moléculaire d'immunoglobulines sériques, causée par la prolifération incontrôlée d'un clone unique de lymphocytes $B$, et constituée soit d'une seule classe de chaîne lourde et d'un seul type de chaîne légère, soit de chaînes légères isolées d'un seul type, soit beaucoup plus rarement de fragments de chaînes lourdes d'une seule classe. Sa présence n'est pas systématiquement synonyme de malignité. Le diagnostic biologique d'une immunoglobuline monoclonale repose sur la réalisation d'une analyse conjointe du sérum et des urines qui vise à affirmer son homogénéité de charge et d'isotypie. Le diagnostic biologique de gammapathie monoclonale ne se limite pas à un seul dosage, mais dépend d'une stratégie raisonnée, utilisant les différents outils diagnostiques que sont l'électrophorèse, qu'elle soit sur gel ou capillaire, l'immunoélectrophorèse ou immunofixation, l'immunosélection, les dosages d'immunoglobulines et de leurs chaînes légères libres, et enfin la recherche de cryoglobuline.

Devant un tableau d'insuffisance rénale aiguë, outre le traitement symptomatique d'épuration, il est important de mettre en route le plus rapidement possible le traitement étiologique. Dans ce contexte, l'affirmation d'une gammapathie monoclonale, parfois suspectée sur l'existence d'un pic à l'électrophorèse, va être capitale. Il faut rappeler qu'une protéine de Bence Jones, ou chaîne légère libre monoclonale sérique, peut parfaitement être indétectable par électrophorèse, qu'elle soit sur gel d'agarose ou capillaire, et prendre en défaut les dosages néphélémétriques [30].

Devant des signes cliniques évocateurs de syndrome d'hyperviscosité (lenteur d'idéation, céphalées, vertige, 


\section{Dossier scientifique}

\section{Figure 1 - Exploration de l'expression des molécules HLA-DR par les lymphocytes T.}
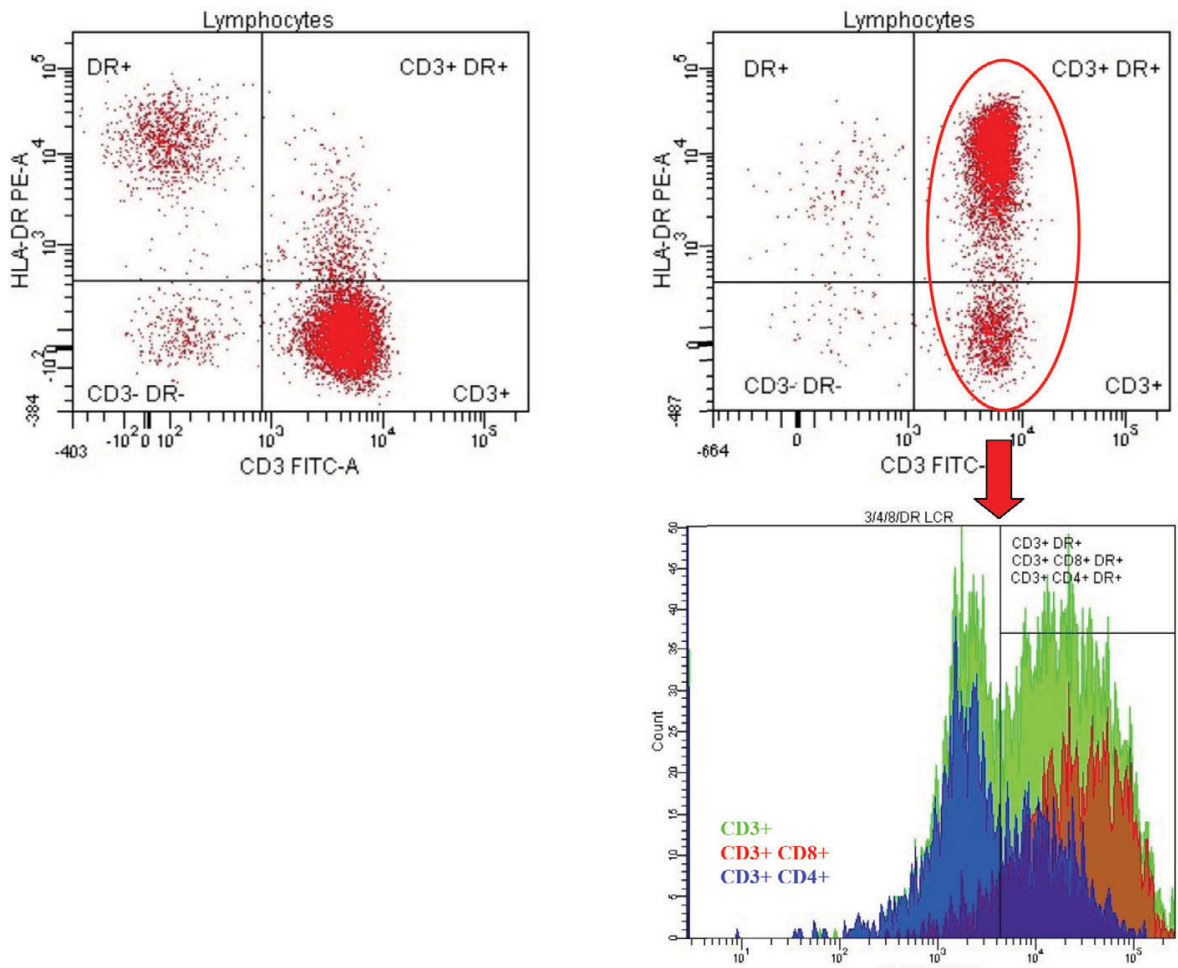

En haut cytogramme confrontant cette expression avec la présence de CD3, marqueur des cellules T; à gauche répartition normale et à droite lors d'un syndrome d'activation macrophagique. En bas, superposition des histogrammes de répartition des lymphocytes T globaux et de leurs sous-populations CD8+ CD4- et CD4+ CD8-, en fonction de leur expression de HLA-DR.

Figure 2 - Histogrammes de répartition des lymphocytes T globaux et de leurs sous-populations CD8+ CD4- et CD4+ CD8-, en fonction de leur expression membranaire des molécules HLA-DR.

Lymphocytes T

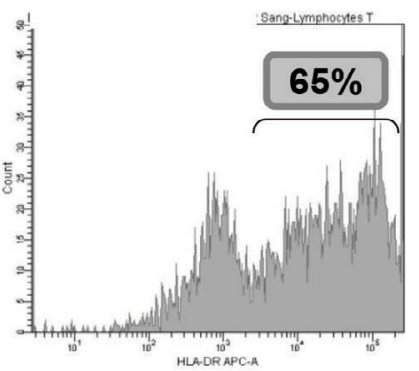

Lymphocytes T

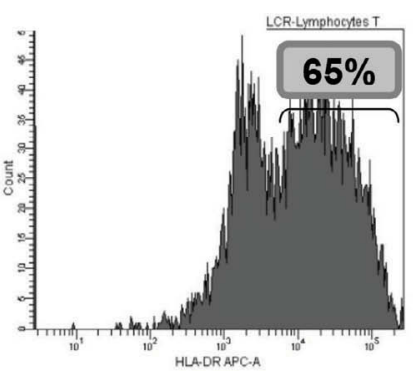

T CD8+4- (75\%)

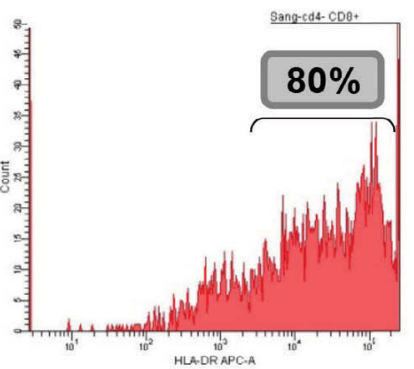

T CD8+4- (42\%)

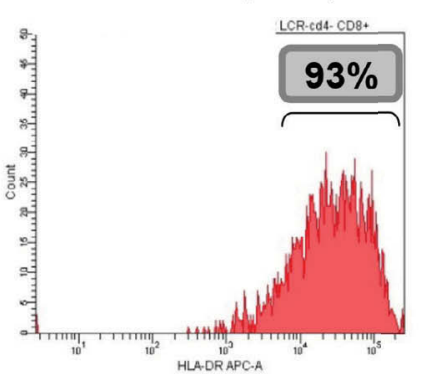

T CD4+8- (22\%)

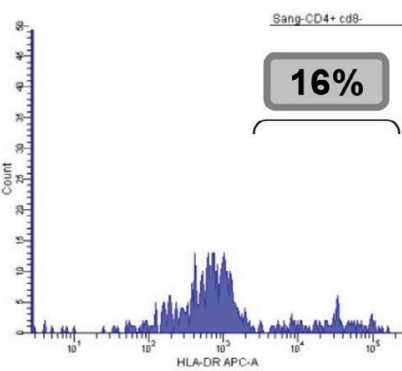

T CD4+8- (55\%)

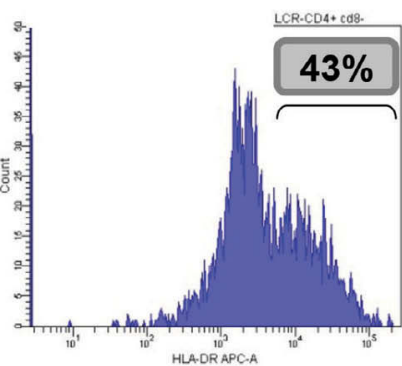


troubles visuels, insuffisance rénale, hémorragie ), outre l'affirmation d'une immunoglobuline monoclonale, le plus souvent d'isotype lgM, selon la procédure décrite ci-dessus, on peut être amené à pratiquer dans la journée une viscosité sérique qui est le meilleur marqueur de suivi de l'efficacité du traitement que sont les échanges plasmatiques.

\subsection{Tests commercialisés, interprétation biologique}

Pour la description de ces techniques, nous renvoyons le lecteur à nos revues précédentes [30, 31]. La part automatisée (immunofixation) des techniques d'identification des gammapathies monoclonales peut être réalisée dans la demi-journée tout comme la recherche de la viscosité sérique. Les dosages pondéraux des immunoglobulines par néphélémétrie ou turbidimétrie, également réalisables dans la demi-journée compléteront ce bilan, quantifiant une éventuelle hypogammaglobulinémie résiduelle, tout en gardant présent à l'esprit que le résultat pour l'isotype de la gammapathie monoclonale est soumis aux réserves imposées par un possible effet de zone [32].

Déclaration d'intérêts: les auteurs déclarent ne pas avoir de conflits d'intérêts en relation avec cet article.

\section{Références}

[1] MHC and transplanation in The HLA complex in biology and medicine, a resource book. Mehra NK (ed) Jaype, New Dehli, 2010:449-576. [2] Jennette JC, Falk RJ, Hu P, et al. Pathogenesis of antineutrophil cytoplasmic autoantibody-associated small-vessel vasculitis. Annu Rev Pathol 2013;8:139-60.

[3] Sayegh J, Poli C, Chevailler A, et al. Emergency testing for antineutrophil cytoplasmic antibodies combined with a dialog-based policy between clinician and biologist: effectiveness for the diagnosis of ANCA-associated vasculitis. Intern Emerg Med 2015;10(3)315-9.

[4] Luxton G, Langham R. ANCA serology in the diagnosis and managment of ANCA-associated renal vasculitis. Nephrology 2008;13:S17-S23.

[5] ANCA fiche 4.9 \& anti-MBG fiche 4.10 , in Guide des analyses en immunologie, indication, critères,de réalisation et limites. @ssim, SFI (eds) Elsevier Masson, Paris, 2014:42-5

[6] Radice A, Bianchi L, Glionna S et al. Clinical evaluation of a rapid immunofluorescence test (IIFT) for diagnosis of ANCA-associated vasculitis $16^{\text {th }}$ international vasculitis and ANCA workshop, Paris, 14-17 avril 2103, communication orale, Presse Med 2013;42(4,cahier 2): A28: 663

[7] Sowa M, Trezzi B, Somma B, et al. Unique technology for the simultaneous screening and confirmation of auoantibodues in emergency situations of rapidly progressive glomerulonephritis $17^{\text {th }}$ international vasculitis and ANCA workshop, Londres, 19-22 avril 2105, communication affichée, Nephron 2015;129(suppl2):P25:118-9

[8] Tur BS, Süldür N, Ataman S, et al. Anti-neutrophil cytoplasmic antibodies in patients with rheumatoid arthritis: clinical, biological, and radiological correlations. Jt Bone Spine Rev Rhum 2004;71(3): 198-202.

[9] Subra JF, Michelet C, Laporte J, et al. The presence of cytoplasmic antineutrophil cytoplasmic antibodies (C-ANCA) in the course of subacute bacterial endocarditis with glomerular involvement, coincidence or association? Clin Nephrol 1998;49(1):15-8.

[10] Beauvillain C, Delneste Y, Renier G, et al. Antineutrophil cytoplasmic autoantibodies: how should the biologist manage them? Clin Rev Allergy Immunol. 2008;35(1-2):47-58.

[11] Poli C, Deguigne PA, Augusto JF, et al. Évaluation de la pratique en urgence de la recherche des anticorps anti-cytplasme des polynucléaires neutrophiles. Rev Fr Lab 2014;464bis:29-35

[12] Lieberman P, Nicklas RA, Oppenheimer J, et al. The diagnosis and management of anaphylaxis practice parameter: 2010 update. J Allergy Clin Immunol 2010;126(3):477-80.e1-42.

[13] Roumier AS, Marin AS et le groupe hypersensibilité de la SFI. Exploration biologique de l'hypersensibilité immédiate. Rev Fr Lab 2002; 341:73-83.

[14] Dosage de la tryptase sérique ou plasmatique fiche 2.1 \& dosage de l,histamine plasmatiquefiche 2.2, in: Guide des analyses en immunologie, indication, critères de réalisation et limites. @ssim, SFI (eds) Elsevier Masson, Paris, 2014:14-6.

[15] Lin RY, Schwartz LB, Curry A, et al. Histamine and tryptase levels in patients with acute allergic reactions: An emergency department-based study. J Allergy Clin Immunol 2000;106(1Pt1):65-71.

[16] Stone SF, Cotterell C, Isbister GK, et al. Elevated serum cytokines during human anaphylaxis: Identification of potential mediators of acute allergic reactions. J Allergy Clin Immunol 2009;124(4):786-92.e4.

[17] Ansari MQ, Zamora JL, Lipscomb MF. Postmortem diagnosis of acute anaphylaxis by serum tryptase analysis. A case report. Am J Clin Pathol 1993;99(1):101-3.

[18] Beauvillain C, Renier G, Jeannin P, et al. Apport des tests de quantification de la libération d'interféron gamma par les lymphocytes $T$ sensibilisés pour le diagnostic des infections tuberculeuses. Rev Fr Lab 2009;410:33-40.

[19] Recommandations HCSP http://www.hcsp.fr/docspdf/avisrapports/hcsp20110701_interferongamma.pdf

[20] Graham SM, Ahmed T, Amanullah F, et al. Evaluation of tuberculosis diagnostics in children: 1 . Proposed clinical case definitions for classification of intrathoracic tuberculosis disease. Consensus from an expert panel. J Infect Dis 2012;205(Suppl2):S199-208.

[21] Basu Roy R, Sotgiu G, Altet-Gómez N, et al. Identifying predictors of interferon- $\gamma$ release assay results in pediatric latent tuberculosis: a protective role of bacillus Calmette-Guerin?:a pTB-NET collaborative study. Am J Respir Crit Care Med 2012;186(4):378-84.

[22] Sollai S, Galli L, de Martino M, et al. Systematic review and metaanalysis on the utility of Interferon-gamma release assays for the diagnosis of Mycobacterium tuberculosis infection in children: a 2013 update. BMC Infect Dis 2014;14(Suppl1):S6.

[23] Test immunologique de dépistage de l'infection tuberculeuse latente par ELISA-IFN-g (Quantiferon ${ }^{\circledR}$ ) fiche 15.1 \& Test immunologique de dépistage de linfection tuberculeuse latente par ELISPOTIFN-g (T-SPOT-TB ${ }^{\circledR}$ ) fiche 15.2, in: Guide des analyses en immunologie, indication, critères de réalisation et limites. @ssim, SFI (eds) Elsevier Masson, Paris, 2014:212-21

[24] Metcalfe JZ, Cattamanchi A, McCulloch CE, et al. Test variability of the QuantiFERON-TB gold in-tube assay in clinical practice. Am J Respir Crit Care Med 2013;187(2):206-11.

[25] Chiacchio T, Petruccioli E, Vanini V, et al. Polyfunctional T-cells and effector memory phenotype are associated with active TB in HIVinfected patients. J Infect 2014;69(6):533-45.

[26] Ongaya A, Huante MB, Mwangi P, et al. Mycobacterium tuberculosis-specific CD8+ T cell recall in convalescing TB subjects with HIV co-infection. Tuberc Edinb Scotl 2013;93(Suppl):S60-5.

[27] Lancioni C, Nyendak M, Kiguli S, et al. CD8+ T cells provide an immunologic signature of tuberculosis in young children. Am J Respir Crit Care Med 2012;185(2):206-12

[28] Janka GE, Lehmberg K. Hemophagocytic syndrome: an up-date. Blood Review 2014;28:135-42

[29] Henter JI, Horne A, Aricó M, et al. HLH-2004: diagnostic and therapeutic guidelines for hemophagocytic lymphohistiocytosis. Pediatr Blood Cancer 2007;48:124-31.

[30] Beauvillain C, Jeannin P, Renier G, et al. Immunoglobulines monoclonales: méthodes diagnostiques en 2011. Rev Fr Lab 2011; 435:55-62.

[31] Lapalus E, Chevailler A. Diagnostic biologique d'une immunoglobuline monoclonale. Rev Fr Lab 2000;327:67-74.

[32] Technique sans traceur: immunoprécipitation, in: Méthode en immunologie. @ssim, SFI (eds) Elsevier Masson, Paris, 2014:25-8. 